\title{
PROCESO DE HORMONIZACIÓN ORGÁNICA, BAJO CONSUMO DE SUSTANCIAS BOTÁNICAS Y ALIMENTOS
}

\section{Organic hormone replacement therapy using botanical substances \& foods}

\author{
María Jose Brizuela LÓPEZ ${ }^{1}$ *
}

\footnotetext{
${ }^{1}$ Universidad Nacional de Córdoba, Facultad de Filosofía y Humanidades, Licenciatura en Antropología, Córdoba, Argentina. *eyulfeuqoir@gmail.com
}

\begin{abstract}
RESUMEN
Nací siendo, según el paradigma médico, varón. Experimenté, como tantos otros niños y niñas, una transición de género conocida como transexualidad. Transité este proceso a partir de una búsqueda personal, favoreciendo cambios hormonales y anatómicos deseados gracias a una alimentación diferencial. Pasados los años, fui diagnosticada con variabilidad intersexual producida por el tratamiento. Transmito en este trabajo la experiencia sobre los cambios somáticos y anatómicos producidos en mi cuerpo a partir del consumo de agentes botánicos y alimentos, para consolidar una terapia de reemplazo / sustitución hormonal orgánica (TRH/TSH). Al compartir esta experiencia, quisiera aportar desde la antropología discusiones biológicas que buscan poner en tensión las construcciones sexo-genéricas que definen masculinidad y feminidad.
\end{abstract}

PALABRAS CLAVE: Hormonización orgánica, Intersexualidad, Transexual, Transgénero, Corporalidad.

\begin{abstract}
I was born being, according to the medical paradigm, male. I experienced, like so many other boys and girls, a gender transition known as transsexuality. I went through this process from a personal search, favoring desired hormonal and anatomical changes thanks to a differential diet. Over the years, I was diagnosed with intersex variability produced by the treatment. In this work I transmit experience on the somatic and anatomical changes produced in my body from the consumption of botanical agents and food, to consolidate an organic hormone replacement / substitution therapy (TRH/TSH). By sharing this experience, I seek to contribute biological discussions from anthropology that seek to put in tension the sex-generic constructions that define masculinity and femininity.
\end{abstract}

KEYWORDS: Intersexuality; hormonization; transexuality.

\section{INTRODUCCIÓN}

Fui nombrada varón y como tal porté por veintiún años un Documento Nacional de Identidad con nombre masculino. Experimenté, como tantos otros niños y niñas, una transición de género conocida como transexualidad y, con el pasar de los años bajo este tratamiento, un desarrollo gonadal diferente conocido como intersexualidad. Dada la falta 
LÓPEZ, M. J. B. Proceso de hormonización orgánica, bajo consumo de sustancias botánicas y alimentos. Ethnoscientia v.6 n.2, especial, 2021. DOI: 10.22276/ethnoscientia.v6i2.380

de aceptación y respeto social sobre la transexualidad, me vi obligada a extraer información de diversas fuentes tales como páginas web, consultas a especialistas en medicina herbal, para experimentar en mi cuerpo cambios hormonales y anatómicos deseados. Fue así, al comprender que es gracias a la alimentación que las proteínas y nutrientes adquiridos logran la reproducción y división celular (meiosis y mitosis), para así desarrollar tejidos y órganos (BROOKER; BERKOWITZ, 2015).

Me vi atraída a investigar diferentes fuentes químicas alimenticias de donde obtener los recursos para desarrollar una reproducción celular con la forma anatómica conocida como femenina, sin recurrir a sustancias farmacológicas. Los saberes populares, los comentarios generales en el popular de la gente, incluyendo los que mi familia tenía sobre las propiedades de algunas plantas ayudaron a este proceso; como también las publicaciones científicas, que tras experimentos en laboratorios, pueden certificar los efectos y propiedades de sustancias botánicas estudiadas. Las publicaciones de la Biblioteca Nacional de Medicina de EE.UU (US National Library of Medicine) demuestran tratamientos tanto orgánicos como químicos, utilizados para combatir el hirsutismo, la hiperplasia suprarrenal congénita o el cáncer de próstata. Éstos tienen como objetivo disminuir la testosterona controlando los receptores de andrógenos (“AR"), los correguladores de AR y otros mecanismos que afectan a la existencia de dichas hormonas en el cuerpo humano (GRANT; RAMASAMY, 2012). Estas investigaciones, como también las del biólogo molecular Humphrey Rawlinson (2010), quien ha publicado diversos artículos en la temática de hormonas y tratamientos orgánicos, han certificado la eficacia del uso de té verde (Camellia sinensis (L.) Kuntze), regaliz (Glycyrrhiza glabra L.), menta (Mentha spicata L.) y otros agentes botánicos para la inhibición de la 5- alfa- reductasa y otros intermediarios en la obtención y producción de testosteronas y andrógenos en el cuerpo.

El presente trabajo tiene como objetivo contar una experiencia sobre cambios somáticos y anatómicos producidos en mi cuerpo a partir de la ingesta de alimentos y agentes botánicos con propiedades estrogénicas y antiandrogénicas, para así consolidar una terapia de reemplazo / sustitución hormonal orgánica. Relato aquí los últimos nueve años que he llevado a cabo esta experiencia, viviendo entre los países Perú, Bolivia, Chile y Argentina. En cada uno de ellos las condiciones de vida, trabajo y residencia variaron, pero dí continuidad a la terapia hormonal adaptándome a las especies botánicas comestibles y los usos y formas de ingesta populares de cada lugar. Al compartir esta experiencia, busco poner en tensión las construcciones disciplinares que definen masculinidad y feminidad como también, las conductas a ellas asociadas, según el aspecto y funcionamiento de los 
LÓPEZ, M. J. B. Proceso de hormonización orgánica, bajo consumo de sustancias botánicas y alimentos. Ethnoscientia v.6 n.2, especial, 2021. DOI: 10.22276/ethnoscientia.v6i2.380

genitales, las hormonas y cromosomas de cada cuerpo. Antaño, estas cuestiones han sido consideradas como exclusivamente "biológicas", mientras, lo referido al género y a la auto percepción de los individuos, como cuestiones de las "ciencias sociales" (GARCÍA GARCÍA, 2003). Al mismo tiempo, diversos estudios biológicos demuestran que las fronteras binarias de sexo, género y cultura, son cada vez más difusas, verificando que a través de una nutrición adecuada los órganos del cuerpo varían su materialidad, influyendo epigenéticamente en la traducción diferencial de la información del ADN y en los procesos de meiosis y mitosis de cada individuo (LOAEZA-LOAEZA; BELTRAN; HERNÁNDEZSOTELO, 2020; McCARTHY et. al, 2009).

\section{METODOLOGÍA}

Durante todos estos años me serví de múltiples disciplinas y saberes para llevar a cabo este proceso. Ingiriendo alimentos, infusiones, extractos/ macerados hidroalcohólicos y realizando los posteriores análisis clínicos de sus efectos hormonales y nutricionales. También me valí de consultas realizadas a curanderas y yerbateras/os, endocrinólogas/os, médicas/os, biólogas/os genetistas, así como en publicaciones científicas que abarcan los campos de la genética y endocrinología transexual e intersexo (CARPENTER, 2018; REISMAN; GOLDSTEIN, 2018). Para la obtención de datos llevé adelante un trabajo de campo etnográfico basado en entrevistas y conversaciones informales con diferentes interlocutoras y en la confección de registros etnográficos de dichos encuentros en cuadernos de campo.

Con las curanderas (las cuales fueron, en su mayoría, mujeres transgénero/travestis/transexuales como también mujeres cisgénero) los lugares de encuentro más frecuentes eran, por lo general, los mercados. En la República del Perú durante los años 2013 y 2015, estos encuentros se llevaron a cabo entre los departamentos y provincias de Lima (Mercados de San Juan de Lurigancho, Surquillo, Barranco y Miraflores) y en Cuzco (Mercado Central de San Pedro y Urubamba); en Bolivia fueron entre los años 2012 y 2013, en los departamentos de La Paz (Mercado de las Brujas), Oruro y Potosí (Mercados centrales); en el caso de las Yerbateras/os, algunas/os aprendices de curanderas/os, los encuentros transcurrieron en Chile (Valparaíso, Santiago de Chile); y en Córdoba, Argentina (Departamento Colón y Capital) durante los años 2015 y 2020. Para el registro de especies botánicas y prácticas culinarias, también tomé en cuenta comentarios y sugerencias de otras mujeres transgénero, transexuales con quienes mantuve conversaciones informales en instancias como el trabajo sexual callejero, reuniones informales, cumpleaños 
LÓPEZ, M. J. B. Proceso de hormonización orgánica, bajo consumo de sustancias botánicas y alimentos. Ethnoscientia v.6 n.2, especial, 2021. DOI: 10.22276/ethnoscientia.v6i2.380

como también en eventos realizados por agrupaciones políticas por los derechos humanos y la visibilidad trans a las cuales asistí en cada uno de los países mencionados durante estos años. La identificación de las muestras vegetales se basó en los nombres vernáculos indicados por mis interlocutoras. En Córdoba (Arg), entre agosto del 2018 y noviembre del 2020, realicé consultas con médicas/os, endocrinólogas/os, quienes continúan acompañándome actualmente en el Hospital Municipal Villa el Libertador Príncipe de Asturias y en el Hospital Córdoba.

La búsqueda bibliográfica fue extensa, pero citare los trabajos más relevantes en la redacción de este trabajo, utilice el motor de búsqueda Google Scholar con las siguientes palabras claves, buscadas por separado: endocrinología, intersexual, transexual, terapias de reemplazo o sustitución hormonal, estrógenos, testosterona, plantas antiandrogénicas, terapias alternativas menopausia, alimentos ricos en Zinc, alimentos ricos en estrógenos, alimentos ricos en testosterona. A su vez, consulté como material de referencia los escritos de Rawlinson (2010), Daphna y MacCarthy (2017) y Curtis (2008).

\section{RESULTADOS}

En total, utilizo y analizo veintiocho especies en mi dieta. El consumo de algunas depende del acceso socioeconómico, en caso de que no sean de la zona o tenga dificultad para encontrarlas en los mercados. Dentro de éstas, considero esenciales las siguientes: Quinua (Chenopodium quinoa Willd), Camote (Ipomoea batatas (L.) Lam), Papas (Solanum tuberosum L.), Yuca o Mandioca (Manihot esculenta Crantz), Zanahoria (Daucus carota L.), Lentejas (Lens culinaris Medik), Soya o Soja (Glicina Max (L.) Merr), Aguaje (Mauritia flexuosa L.f), Moras (Morus alba L.), Lino (Linum usitatissimum L.), Zapallo (Cucurbita spp.), Maíz (Zea mays L.), Té verde (Camellia sinensis (L.) Kuntze), Menta (Mentha alaica Boriss), Regaliz (Glycyrrhiza bucharica Regel), Doradilla (Anemia australis (Mickel) M. Kessler \& A.R. Sm.), Angélica (Angelica archangelica L.), Stevia (Stevia rebaudiana (Bertoni) Bertoni) y Salvia (Salvia officinalis L.). Las plantas mencionadas son las que más he usado para este proceso, afectando el estado de mi sangre y conformando cambios anatómicos que se detallan en un apartado específico más abajo; éstos fueron contundentes y aún me encuentro atravesando estos procesos de transición. Se recomienda comer de una especie libre de agrotóxicos. 
LÓPEZ, M. J. B. Proceso de hormonización orgánica, bajo consumo de sustancias botánicas y alimentos. Ethnoscientia v.6 n.2, especial, 2021. DOI: 10.22276/ethnoscientia.v6i2.380

Tabla 1: Listado de especies libres de agro tóxicos ricas en estrógenos, isoflavonas y progesterona. Información recopilada durante el trabajo de campo por la autora. Fecha: 2012 - 2021.

\begin{tabular}{|c|c|c|c|c|}
\hline $\mathbf{N}^{\mathbf{0}}$ & $\begin{array}{l}\text { Especie botánica } \\
\text { "Nombre común" }\end{array}$ & $\begin{array}{l}\text { Origen cultural } \\
\text { (origen } \\
\text { biogeográfico) }\end{array}$ & Fuente & Observaciones \\
\hline \multicolumn{5}{|c|}{ Ricas en fitoestrógenos, progesterona e isoflavonas: } \\
\hline 1 & $\begin{array}{l}\text { Glycine max } \\
\text { (L.) Merr. "Soja" }\end{array}$ & Asia. (Exótica) & $\begin{array}{l}\text { Curanderas y } \\
\text { otras transexuales, } \\
\text { Rawlinson (2010) }\end{array}$ & $\begin{array}{l}\text { Se recomienda comer de una } \\
\text { especie libre de agrotóxicos }\end{array}$ \\
\hline 2 & $\begin{array}{l}\text { Mauritia flexuosa } \\
\text { L.f. "Aguaje" }\end{array}$ & $\begin{array}{l}\text { Amazonas, Brasil, } \\
\text { Perú, Bolivia. } \\
\text { (Exótica) }\end{array}$ & $\begin{array}{l}\text { Curanderas Perú y } \\
\text { Bolivia }\end{array}$ & $\begin{array}{l}\text { Esta especie solo se consigue en } \\
\text { esos países hasta donde investigue, } \\
\text { quizás se consiga en otros. Es } \\
\text { difícil conseguir esta especie en } \\
\text { zonas que no cumplan con ciertas } \\
\text { propiedades en la tierra y humedad } \\
\text { en el clima. }\end{array}$ \\
\hline 3 & $\begin{array}{l}\text { Daucus carota } \mathrm{L} . \\
\text { "Zanahoria" }\end{array}$ & $\begin{array}{l}\text { Asia, Europa. } \\
\text { (Exótica) }\end{array}$ & Rawlinson (2010) & $\begin{array}{l}\text { En su forma de alimento crudo o } \\
\text { cocido. }\end{array}$ \\
\hline 4 & $\begin{array}{l}\text { Lens culinaris } \\
\text { Medik. "Lentejas", }\end{array}$ & $\begin{array}{l}\text { Mediterráneo. } \\
\text { (Exótica) }\end{array}$ & Rawlinson (2010) & $\begin{array}{l}\text { En su forma de alimento cocido o } \\
\text { en brotes. }\end{array}$ \\
\hline 5 & $\begin{array}{l}\text { Linum } \\
\text { usitatissimum L. } \\
\text { "Linaza" }\end{array}$ & $\begin{array}{l}\text { Mediterráneo. } \\
\text { (Exótica) }\end{array}$ & $\begin{array}{l}\text { Curanderas, } \\
\text { Yerbateras/os, } \\
\text { Rawlinson } \\
(2010) .\end{array}$ & $\begin{array}{l}\text { La mejor forma, aparte de ingerir } \\
\text { el aceite puro comestible, es beber } \\
\text { la baba que se genera al remojar } \\
\text { las semillas durante veinticuatro } \\
\text { horas o hirviéndolas en una } \\
\text { cantidad que duplica o supera su } \\
\text { tamaño en agua. }\end{array}$ \\
\hline 6 & $\begin{array}{l}\text { Dioscorea spp. } \\
\text { "Name, papas" }\end{array}$ & $\begin{array}{l}\text { África, Asia, } \\
\text { América. (Exótica) }\end{array}$ & $\begin{array}{l}\text { Curanderas del } \\
\text { Perú y Bolivia, } \\
\text { Rawlinson } \\
(2010) .\end{array}$ & En su forma de alimento cocido. \\
\hline 7 & $\begin{array}{l}\text { Manihot esculenta } \\
\text { Crantz } \\
\text { "Yuca, mandioca" }\end{array}$ & $\begin{array}{l}\text { América del Sur. } \\
\text { (Exótica) }\end{array}$ & $\begin{array}{lr}\text { Curanderas } & y \\
\text { otras } & \text { transexuales } \\
\text { del Perú } \quad y \\
\text { Bolivia. }\end{array}$ & $\begin{array}{l}\text { En sus formas populares de } \\
\text { alimento cocido. }\end{array}$ \\
\hline 8 & $\begin{array}{l}\text { Ipomoea batatas } \\
\text { (L.) Lam "Camote" }\end{array}$ & $\begin{array}{l}\text { Península de } \\
\text { Yucatán, México. } \\
\text { (Exótica) }\end{array}$ & $\begin{array}{lr}\text { Curanderas } & y \\
\text { otras } & \text { transexuales } \\
\text { del Perú } \quad y \\
\text { Bolivia. }\end{array}$ & $\begin{array}{l}\text { Comer en forma de alimento } \\
\text { cocido. }\end{array}$ \\
\hline 9 & $\begin{array}{l}\text { Chenopodium } \\
\text { quinoa Willd. } \\
\text { "Quinoa" }\end{array}$ & $\begin{array}{l}\text { América del Sur. } \\
\text { (Nativa) }\end{array}$ & $\begin{array}{l}\text { Curanderas del } \\
\text { Perú y Bolivia. }\end{array}$ & $\begin{array}{l}\text { Comer en su forma popular de } \\
\text { alimento cocido como cualquier } \\
\text { legumbre. }\end{array}$ \\
\hline 10 & $\begin{array}{l}\text { Condalia } \\
\text { microphylla Cav. } \\
\text { "Piquillín" }\end{array}$ & $\begin{array}{l}\text { Argentina. } \\
\text { (Endémica) }\end{array}$ & Yerbateras/os. & $\begin{array}{l}\text { Comer sus frutos de manera } \\
\text { directa, mermeladas, arropes o en } \\
\text { macerados hidroalcohólicos. }\end{array}$ \\
\hline 11 & $\begin{array}{l}\text { Sarcomphalus } \\
\text { mistol (Griseb.) } \\
\text { Hauenschild } \\
\text { "Mistol" }\end{array}$ & $\begin{array}{l}\text { América del Sur. } \\
\text { (Nativa) }\end{array}$ & Yerbateras/os. & $\begin{array}{l}\text { Comer sus frutos en sus formas } \\
\text { populares: arrope, infusión, } \\
\text { macerados hidroalcohólicos o café. }\end{array}$ \\
\hline 12 & $\begin{array}{l}\text { Morus alba L. } \\
\text { "Mora" }\end{array}$ & Pérsico. (Exótica) & Yerbateras/os. & $\begin{array}{l}\text { Consumir sus hojas secas en } \\
\text { infusión, y sus frutas crudas o en } \\
\text { mermelada. }\end{array}$ \\
\hline 13 & $\begin{array}{l}\text { Ullucus tuberosus } \\
\text { Caldas "Olluco" }\end{array}$ & $\begin{array}{l}\text { América del Sur. } \\
\text { (Nativa) }\end{array}$ & $\begin{array}{l}\text { Curanderas y } \\
\text { otras transexuales } \\
\text { del Perú }\end{array}$ & $\begin{array}{l}\text { Solo pude conseguirla en Perú y } \\
\text { Bolivia. Comiéndola cocida en las } \\
\text { comidas. }\end{array}$ \\
\hline 14 & $\begin{array}{l}\text { Curcubitas sp. } \\
\text { (todas las especies } \\
\text { de "calabazas"y } \\
\text { "zapallos") }\end{array}$ & América. (Nativa) & $\begin{array}{l}\text { Curanderas, } \\
\text { transexuales y } \\
\text { Yerbateras. }\end{array}$ & $\begin{array}{l}\text { En forma de alimento cocido o } \\
\text { rallado y crudo. }\end{array}$ \\
\hline
\end{tabular}


LÓPEZ, M. J. B. Proceso de hormonización orgánica, bajo consumo de sustancias botánicas y alimentos. Ethnoscientia v.6 n.2, especial, 2021. DOI: 10.22276/ethnoscientia.v6i2.380

\begin{tabular}{|c|c|c|c|c|}
\hline 15 & $\begin{array}{l}\text { Foeniculum } \\
\text { vulgare Mill. } \\
\text { "Hinojo" }\end{array}$ & $\begin{array}{l}\text { Mediterráneo. } \\
\text { (Exótica) }\end{array}$ & $\begin{array}{l}\text { Curanderas, } \\
\text { Yerbateras/os, } \\
\text { Rawlinson (2010) } \\
\text { y otras } \\
\text { transexuales. }\end{array}$ & En forma de infusión. \\
\hline 16 & $\begin{array}{l}\text { Prosopis spp. } \\
\text { "Algarrobo" }\end{array}$ & $\begin{array}{l}\text { América del Sur. } \\
\text { (Nativa) }\end{array}$ & Yerbateras/os. & $\begin{array}{l}\text { En sus formas populares de arrope } \\
\text { y/o harina en panificación. Todos } \\
\text { los tipos de Algarrobo nativos de } \\
\text { América fueron utilizados. }\end{array}$ \\
\hline 17 & $\begin{array}{l}\text { Zea mays L. } \\
\text { "Choclo, maíz" }\end{array}$ & América. (Exótica) & $\begin{array}{l}\text { Curanderas, } \\
\text { Yerbateras/os y } \\
\text { otras transexuales. }\end{array}$ & $\begin{array}{l}\text { En sus formas tradicionales } \\
\text { comestibles, especialmente la } \\
\text { especie de Zea Mays var. indentata } \\
\text { "Maíz morado". }\end{array}$ \\
\hline 18 & $\begin{array}{l}\text { Salvia officinalis L. } \\
\text { "Salvia" }\end{array}$ & $\begin{array}{l}\text { Mediterráneo. } \\
\text { (Exótica) }\end{array}$ & $\begin{array}{l}\text { Curanderas, } \\
\text { Yerbateras/os, } \\
\text { Rawlinson }(2010)\end{array}$ & $\begin{array}{l}\text { Su forma más óptima es en la de } \\
\text { extracto/macerado } \\
\text { hidroalcohólico. }\end{array}$ \\
\hline 19 & $\begin{array}{l}\text { Actaea racemosa } \\
\text { L. "Cimicifuga" }\end{array}$ & $\begin{array}{l}\text { Norte América. } \\
\text { (Exótica) }\end{array}$ & $\begin{array}{l}\text { Yerbateras/os, } \\
\text { Rawlinson } \\
(2010) \text {. }\end{array}$ & $\begin{array}{l}\text { En extracto/macerado } \\
\text { hidroalcohólico. }\end{array}$ \\
\hline 20 & $\begin{array}{l}\text { Angelica } \\
\text { archangelica } \mathrm{L} . \\
\text { "Angélica" }\end{array}$ & Norte de Europa. & $\begin{array}{l}\text { Yerbateras/os, } \\
\text { Rawlinson } \\
(2010) .\end{array}$ & $\begin{array}{l}\text { En extracto/macerado } \\
\text { hidroalcohólico. }\end{array}$ \\
\hline 21 & $\begin{array}{l}\text { Anemia australis } \\
\text { (Mickel) M. } \\
\text { Kessler \& A.R. } \\
\text { Sm. } \\
\text { "Doradilla" }\end{array}$ & Argentina. (Nativa) & $\begin{array}{l}\text { Yerbateras/os de } \\
\text { Córdoba. }\end{array}$ & $\begin{array}{l}\text { En extracto/macerado } \\
\text { hidroalcohólico. }\end{array}$ \\
\hline \multicolumn{5}{|c|}{ Con propiedades antiandrogénicas: } \\
\hline 22 & $\begin{array}{l}\text { Mentha spicata L. } \\
\text { "Hierba buena" }\end{array}$ & $\begin{array}{l}\text { Mediterránea. } \\
\text { (Exótica) }\end{array}$ & $\begin{array}{l}\text { Curanderas, } \\
\text { yerbateras/os, } \\
\text { Rawlinson }(2010) \\
\end{array}$ & $\begin{array}{l}\text { En infusiones durante todo el día, } \\
\text { y /o en extracto/macerado } \\
\text { hidroalcohólico. }\end{array}$ \\
\hline 23 & $\begin{array}{l}\text { Passiflora } \\
\text { incarnata } \mathrm{L} .\end{array}$ & $\begin{array}{l}\text { México, Norte } \\
\text { América. (Exótica) }\end{array}$ & $\begin{array}{l}\text { Yerbateras/os, y } \\
\text { otras transexuales } \\
\text { de Chile. }\end{array}$ & $\begin{array}{l}\text { En infusiones o extracto/macerado } \\
\text { hidroalcohólico. }\end{array}$ \\
\hline 24 & $\begin{array}{l}\text { Stevia rebaudiana } \\
\text { (Bertoni) Bertoni } \\
\text { "Ka'a he'e" }\end{array}$ & $\begin{array}{l}\text { Paraguay y Sur de } \\
\text { Brasil. (Nativa) }\end{array}$ & $\begin{array}{l}\text { Yerbateras/os, y } \\
\text { otras transexuales } \\
\text { de Chile. }\end{array}$ & $\begin{array}{l}\text { En infusiones durante todo el día, } \\
\text { y/o en extracto/macerado } \\
\text { hidroalcohólico. }\end{array}$ \\
\hline 25 & $\begin{array}{l}\text { Lavandula spp. } \\
\text { "Lavanda" }\end{array}$ & $\begin{array}{l}\text { Región } \\
\text { macaronésica. } \\
\text { (Exótica) }\end{array}$ & $\begin{array}{l}\text { Yerbateras/os y } \\
\text { otras transexuales } \\
\text { de Chile. }\end{array}$ & $\begin{array}{l}\text { En infusiones y } \\
\text { extractos/macerados } \\
\text { hidroalcohólicos. }\end{array}$ \\
\hline 26 & $\begin{array}{l}\text { Camellia sinensis } \\
\text { (L.) Kuntze } \\
\text { "Té verde" }\end{array}$ & Asia. & $\begin{array}{l}\text { Yerbateras/os, } \\
\text { otras transexuales, } \\
\text { Rawlinson (2010) }\end{array}$ & $\begin{array}{l}\text { En infusiones durante todo el día. } \\
\text { (aun no la ingerí su forma de } \\
\text { extracto/macerado } \\
\text { hidroalcohólico) }\end{array}$ \\
\hline 27 & $\begin{array}{l}\text { Glycyrrhiza glabra } \\
\text { L. Regaliz }\end{array}$ & $\begin{array}{l}\text { África, Europa, } \\
\text { Asia. (Exótica) }\end{array}$ & Rawlinson (2010) & $\begin{array}{l}\text { En extracto/macerado } \\
\text { hidroalcohólico. Se advierte de } \\
\text { componentes estrogénicos además } \\
\text { de antiandrogénicos. }\end{array}$ \\
\hline 28 & $\begin{array}{l}\text { Serenoa repens } \\
\text { (W. Bartram) } \\
\text { Small }\end{array}$ & América. & $\begin{array}{l}\text { Yerbateras/os, } \\
\text { Rawlinson (2010) }\end{array}$ & $\begin{array}{l}\text { En extracto/macerado } \\
\text { hidroalcohólico. }\end{array}$ \\
\hline
\end{tabular}

Hasta aquí, comparto la lista de especies probadas en estos nueve años de investigación, los usos y recomendaciones. Durante algunos años, me vi limitada a una asistencia endocrinológica para seguir de manera clínica este proceso. Gracias a la aprobación, en el año 2012, de la Ley de Identidad de Género en Argentina (N. ${ }^{\circ}$ 26.743), 
LÓPEZ, M. J. B. Proceso de hormonización orgánica, bajo consumo de sustancias botánicas y alimentos. Ethnoscientia v.6 n.2, especial, 2021. DOI: 10.22276/ethnoscientia.v6i2.380

que establece el derecho a acceder a tratamientos hormonales e intervenciones quirúrgicas totales o parciales para adecuar el cuerpo a la identidad elegida (Art. 11), pude realizarme análisis de sangre y tener un seguimiento con especialistas en centros médicos. De esta manera, los últimos estudios hormonales realizados certifican la eficacia de este tratamiento como terapia hormonal, debido a que el resultado de los niveles de hormonas en mi cuerpo son los esperados para una persona bajo suministro farmacológico de estradiol e inhibidores de testosterona.

\subsection{Cambios corporales}

Como dije anteriormente, los cambios somáticos y anatómicos registrados fueron importantes y contundentes; aún sigo transitando con gusto y admiración procesos biológicos. A continuación detallo los cambios registrados:

Disminución del vello corporal de las zonas del tipo AR como los hombros, cuello, espalda, rostro, espalda y busto principalmente, importantes cambios en los olores corporales, rotación ligera de la cadera, mayor resistencia en uñas y cabello, aumento y re distribución de grasa, ligeros cambios en los ángulos del rostro, ligeros cambios en la percepción visual de la distancia y la nitidez de los objetos, disminución de la oleosidad del cuero cabelludo y rostro, audición y olfato ligeramente agudizados, desarrollo mamario y secreción de leche materna, cambios en la composición del semen tales como pérdida del color y textura liviana.

Cíclicamente, experimento una sensibilidad emocional alta, como también aumento de la sensibilidad de tipo erógena en zonas corporales como la aureola de los pezones, el pliegue labio escrotal, el pubis y la zona baja del ombligo, el escroto concentra una humedad y oleosidad atípicas, la piel de éste es tornasolada y mucho más oscura en la línea escrotal que divide los testículos; recientemente esta línea se hunde, generando nuevos placeres y formas de goce sexual, como también, desde agosto del año 2018, experimento un sangrado intermitente con uno o dos días de duración cada mes, liberado en la orina. Advirtiendo, por parte del equipo médico con quienes me atiendo, ya en mi adultez, el "despertar" de una variante intersexual que posiblemente siempre estuvo ahí.

Pude experimentar como los alimentos ricos en Zinc cumplen una función importante en la reproducción de las células de ciertos tejidos como los testículos, ya que es este mineral es el que habilita la procreación de testosterona y del desarrollo gonadal conocido como masculino (SALGUEIRO et. al, 2004). Algunos de los alimentos ricos en este mineral son: el gluten de refinados, arroz blanco refinado, harina de trigo blanca 
refinada, carnes rojas y derivados, maní, levaduras, huevo, entre otros; y son, curiosamente, propios de la alimentación europea injertada en Argentina. Su consumo cumple una función fundamental en elevar los niveles de testosterona en el cuerpo. Con el consumo de alimentos ricos en Zinc, en periodos donde los ingerí como única fuente nutricional, he registrado que mis venas se ensancharon, el vello corporal de las zonas AR aumento y con él, el acné. Los senos disminuyen su tamaño y erogeneidad, la alopecia androgénica es más factible, pues generaron un exceso de oleosidad en la frente y cuero cabelludo. Al disminuir su consumo, el tamaño y densidad de los testículos disminuye, la línea escrotal se oscurece; en mi caso, esta línea se hundió generando un nuevo placer sexual, la textura del escroto se ha vuelto húmeda, ha disminuido el vello en esta zona y se ha generado un sangrando periódico en la orina, similar a una menstruación. Médicos con quienes me asesoro han interpretado este sangrado como signo de intersexualidad, dado que no presento infecciones ni patologías, visualizándose en ecografías un posible remanente uterino, el cual se ve estimulado por este tratamiento. Se trata de un asunto que precisa seguir siendo investigado en tanto adquiere especial relevancia para problematizar construcciones sexo-genéricas binarias.

\section{CONCLUSIONES}

He compartido aquí los resultados tanto de mis investigaciones y preguntas como de mi experiencia corporal en la transición conocida en el sentido común como de varón a mujer (MTF siglas en inglés) producida por el consumo de sustancias botánicas y alimentos. La experiencia relatada en este escrito muestra que las prácticas alimenticias son también formas en las que se crean los cuerpos. A partir de este análisis se plantea que existen plantas que son factibles de pensar/emplear para terapias de reemplazo y sustitución hormonal de manera orgánica. Con estas prácticas y estrategias alimenticias se configuran cambios anatómicos propios del proceso de transición sexo-genérica.

Por todo lo antedicho, este trabajo propone pensar a las corporalidades como construcciones y no como "destinos", procesos y elecciones que se dan durante toda la vida de un individuo. Estos dilemas que parecen personales, pero que son sociales, no son otra cosa que formas de cuestionar las fronteras difusas entre naturaleza y cultura y la errónea concepción binaria de la biología sexo-genérica. Dado que mi intersexualidad es resultado de este proceso de hormonización bajo el consumo diferencial de alimentos y agentes botánicos y no un diagnóstico hecho al nacer, pienso: ¿Será posible interpretar la intersexualidad como una posibilidad de todo cuerpo humano, similar a un "alelo" recesivo 
LÓPEZ, M. J. B. Proceso de hormonización orgánica, bajo consumo de sustancias botánicas y alimentos. Ethnoscientia v.6 n.2, especial, 2021. DOI: 10.22276/ethnoscientia.v6i2.380

o dominante, en lugar de una patología fundada en estigmas culturales que pretenden justificar un binarismo natural de la especie humana?

\section{REFERENCIAS BIBLIOGRÁFICAS}

ARGENTINA. Ley nº 26.743/2012. Promulgada el 23 de mayo de 2012. Establécese el derecho a la identidad de género de las personas. Disponible en: http://servicios.infoleg.gob.ar/infolegInternet/anexos/195000-199999/197860/norma.htm. Accedido en: 26 mar. 2021.

BROOKER, A.; BERKOWITZ, K. The roles of cohesins in mitosis, meiosis, and human health and disease. In: Noguchi E., Gadaleta M. (eds) Cell Cycle Control. Methods in Molecular Biology (Methods and Protocols), New York: Humana Press, 2014. vol 1170, pp 229-266. https://doi.org/10.1007/978-1-4939-0888-2_11

CARPENTER, M. Intersex Variations, Human Rights, and the International Classification of Diseases. Health Hum Rights, Boston, v. 20, n. 2, 205-214, 2018 dec. Disponible en: www.ncbi.nlm.nih.gov/pmc/articles/PMC6293350/. Accedido en: 17 dic. 2020.

CURTIS, H. et. al. Biología. 7ma edición. Buenos Aires: Médica Panamericana, 2008. $1160 \mathrm{p}$.

DAPHNA, J.; McCARTHY, M M. Incorporating Sex As a Biological Variable in Neuropsychiatric Research: Where Are We Now and Where Should We Be? Neuropsychopharmacology, London, v. 42, n. 2, 379-385. 2017 Jan. Disponible en: https://pubmed.ncbi.nlm.nih.gov/27240659/. Accedido en: 19 ene. 2021.

GARCÍA GARCÍA, E. Neuropsicología y género. Revista de la Asociación Española de Neuropsiquiatría, Madrid, n. 86, 7-18, 2003 jun. Disponible en: http://scielo.isciii.es/scielo.php?script=sci_arttext\&pid=S0211-57352003000200002.

Accedido en: 19 ene 2021.

GRANT, P.; RAMASAMY, S. An Update on Plant Derived Anti-Androgens. Int J Endocrinol Metab. Theran, v. 20, n. 2, 497-502, 2012 Apr. Disponible en:

www.ncbi.nlm.nih.gov/pmc/articles/PMC3693613/. Accedido en: 17 dic 2020.

LOAEZA-LOAEZA, J., BELTRÁN, A.; HERNÁNDEZ SOTELO, D. DNMTs and Impact of CpG Content, Transcription Factors, Consensus Motifs, 1ncRNAs, and Histone Marks on DNA Methylation. Genes, Basel, v. 11, n. 11, 1336, 2020 nov. Disponible en: www.ncbi.nlm.nih.gov/pmc/articles/PMC7696963/. Accedido en: 19 ene. 2021.

McCARTHY, M. et al. The epigenetics of Sex Differences in the Brain. Journal of Neuroscience, Washington, v. 29, n. 41, 12815-12823, 2009 oct. Disponible en: www.jneurosci.org/content/29/41/12815/tab-article-info Accedido en: 19 ene. 2021.

RAWLINSON, H. Tratamiento natural de la sexualidad. Testosterona y estrógenos. Primera edición. Madrid: Editorial Dilema, 2010, 266p. 
LÓPEZ, M. J. B. Proceso de hormonización orgánica, bajo consumo de sustancias botánicas y alimentos. Ethnoscientia v.6 n.2, especial, 2021. DOI: 10.22276/ethnoscientia.v6i2.380

REISMAN, T.; GOLDSTEIN, Z. Case Report: Induced Lactation in a Transgender Woman. Transgender Health, Chicago, v. 3, n. 1, 24-26, 2018 dic. Disponible en: www.liebertpub.com/doi/10.1089/trgh.2017.0044. Accedido en: 17 dic 2020.

SALGUEIRO, M J. et al. Deficiencia de zinc en relación con el desarrollo intelectual y sexual. Rev Cubana Salud Pública, Ciudad de La Habana, v. 30, n. 2, 2004 jun. Disponible en: http://scielo.sld.cu/scielo.php?script=sci_arttext\&pid=S086434662004000200007\&lng=es\&nrm=iso. Accedido en:19 ene 2021. 